Int. J. Dev. Biol. 61: 311-318 (2017)

doi: $10.1387 / \mathrm{ijdb} .160275 \mathrm{as}$

\title{
Loss of thrombospondin reveals a possible role for the extracellular matrix in chordotonal cap cell elongation
}

\author{
RINA T. GREENBLATT BEN-EL, ABEER HASSAN and ADI SALZBERG* \\ Department of Genetics and Developmental Biology, The Rappaport Faculty of Medicine and Research Institute, \\ Technion-Israel Institute of Technology, Haifa, Israel
}

\begin{abstract}
In the Drosophila larva, major proprioceptive input is provided to the brain by subepidermal stretch receptors called chordotonal organs (ChO). Similarly to the body wall muscle that needs to be attached on both of its sides to the larval exoskeleton in order to generate movement, the sensory unit of a $\mathrm{ChO}$ must be stably anchored to the cuticle on both of its sides in order to sense the relative displacement of body parts. Through an RNAi screen we have identified thrombospondin (Tsp), a secreted calcium binding glycoprotein, as a critical component in the anchoring of ChOs to the cuticle. We show that the Tsp protein starts to accumulate in the extracellular matrix (ECM) surrounding the ChO attachment cells towards the end of embryogenesis and that it becomes highly concentrated at the attachment junction during larval stages. In the absence of Tsp, the ChO's accessory cells fail to form a stable junction with their epidermal attachment cells and organ integrity is not maintained. Tsp is a known player in the establishment of the myotendinous junctions in both invertebrates and vertebrates. Thus, our findings extend the known similarities between muscle-attachment and $\mathrm{ChO}$-attachment cells. In addition to its role in establishing the ChO attachment junctions, Tsp was found to affect ligament cell migration and cap cell elongation. Most interestingly, the Tsp protein was found to decorate the $\mathrm{ChO}$ cap cells along their entire length, suggesting that the elongated cap cells are supported by the ECM to which they attach via integrin-based, Tsp-dependent, adhesion plaques. The ECM enwrapping the cap cells is probably important for keeping the cap cells fasciculate and may also provide mechanical support that allows the extremely elongated cells to maintain tension.
\end{abstract}

KEY WORDS: chordotonal organ, morphogenesis, cell adhesion, ECM

\section{Introduction}

The chordotonal organs (ChO) of a fruit fly larva function as proprioceptive stretch receptors that translate mechanical stimuli into neuronal impulses that allow coordinated larval locomotion (Caldwell et al., 2003). There are several subtypes of ChOs that differ from each other in the types and numbers of cells that constitute them, but they all contain a similar sensory unit that is composed of a ciliated neuron and a specialized glial cell called the scolopale cell. In order to function as a stretch receptor, the sensory unit must be attached on both of its sides to accessory cells that anchor it to the epidermis/cuticle. Cuticle deformation due to muscle contractions leads to relative displacement of the two attachment points. Such mechanical stimulus leads to the opening of TRPV channels at the tip of the dendrite and the elicitation of a signal that is transduced to the brain (Gong et al., 2004; Kim et al., 2003).
Most of our knowledge about the structure of larval ChOs comes from studies focused on the most prominent organ - the lateral pentascolopidial (LCh5) organ. The LCh5 organ contains five sensory units, each of which is stretched between a cap cell at the dendritic side and a ligament cell at the axonal side of the neuron. The cap and the ligament cells of all five organs are anchored to the cuticle by two cap-attachment (CA) cells (Ghysen and Dambly-Chaudiere, 1989; Matthews et al., 1990) and one ligament-attachment cell (LA), respectively (Inbal et al., 2004). During larval growth the CA and LA cells of the LCh5 organs increase in size dramatically and extend numerous tubulin-rich extensions (Halachmi et al., 2016). These morphological changes that enlarge the contact area between

Abbreviations used in this paper: ChO, chordotonal organ; ECM, extra cellular matrix; Tsp, thrombospondin.

*Address correspondence to: Adi Salzberg. Department of Genetics and Developmental Biology. Rappaport Faculty of Medicine, Technion P.O.Box 9649, Haifa 3109601, Israel. Tel.: (+972)-4-8295227 Fax: (+972)-4-8295225. E-mail: adis@technion.ac.il - web: http://www.rappinst.com/Rappaport

- (D) http://orcid.org/0000-0002-0427-9809

Accepted: 10 August, 2016.

ISSN: Online 1696-3547, Print 0214-6282 
the CA and LA cells and their attachment substrate are probably important for their ability to resist the increasing mechanical forces imposed upon them as the larva grows.

Many lines of similarity can be drawn between the patterning of the movement-generating system (muscles and tendons) and the movement-sensing system (chordotonal organs). One of these parallels is the dependence of both systems on stable anchoring to the exoskeleton for proper functioning. In the movement-generating system, epidermal attachment cells that differentiate as tendon cells anchor the muscles to the cuticle. Similarly, in the movementsensing system epidermal attachment cells differentiate as $\mathrm{ChO}$ attachment cells (LA and CA) that anchor the cap and ligament cells to the cuticle. We have previously shown that both systems use similar patterning mechanisms that depend upon the activity of the transcription factor Stripe (Sr) for coordinated patterning of the muscles and their corresponding tendons as well as for the ChOs and their corresponding attachment cells (Klein etal., 2010; reviewed in Hasson et al., 2017). Studies have shown that muscles attach to tendon cells via integrin-rich hemiadherens junctions (Prokop et al., 1998); however, the attachment of ligament and cap cells to their corresponding attachment cells has not been characterized in depth. Nevertheless, we have shown that the contact area between the CA and cap cells, and between the LA and ligament cells, displays a high concentration of integrin linked kinase (ILK), indicating an abundance of integrin-based connections between the cells (Halachmi et al., 2016).

In an attempt to identify the critical components of the ChOspecific attachment machinery we have initiated an RNAi screen (unpublished data), in which RNAi transgenes directed against extra cellular matrix (ECM)-related candidate genes were expressed specifically within cells of the ChO lineage and their neighboring cells in the posterior compartment of the segment. The ChOs were visualized at the $2^{\text {nd }}$ instar larval stage using $\mathrm{ChO}$-specific fluorescent markers (Halachmi et al., 2016). One of the genes identified in the screen as being essential for the proper attachment of the ChO cap cells to the CA cells was thrombospondin ( $t s p$ ), which encodes for a secreted calcium binding glycoprotein that incorporates into the ECM (Adams et al., 2003; Adolph, 2001). Further analysis of tsp mutant embryos revealed the involvement of $t s p$ in terminal differentiation and migration of ligament cells, a phenotype reminiscent of the phenotype of stripe (sr) mutant embryos (Klein et al., 2010). Characterization of Tsp protein distribution in embryos and larvae revealed that Tsp becomes evident in the ECM surrounding the $\mathrm{ChO}$ attachment cells in very late embryonic stages. The distribution of Tsp changes dramatically with organ maturation and it becomes evident in the ECM surrounding additional ChO cells in larval stages. The most surprising observation was the pattern of distribution of Tsp protein along the cap cells, which suggests for the first time that in addition to the integrin/Tsp-dependent attachment of the cap cells to the CA cells, the cap cells must also form integrin/Tsp-dependent adhesion plaques along their entire length. Phenotypic analysis of tsp mutant embryos points to a possible role of this lateral cell-long ECM support in cap cell elongation.

\section{Results}

\section{Thrombospondin is required for proper attachment of larval lateral chordotonal organs}

Early aspects of embryonic ChO development have been studied quite extensively (e.g. Brewster and Bodmer, 1995; Inbal et al., 2003; Jarman et al., 1993), nonetheless our knowledge about the genetic basis of later aspects of morphogenesis and attachment of these organs is very sparse. To start filling in the gaps in our knowledge regarding late morphogenetic events, we havegenerated transgenic flies with fluorescently labeled ChOs that allow easy visualization and phenotypic analyses of ChOs in post-embryonic stages (Halachmi et al., 2016). These new reporters have been recombined to two gal4 drivers, ato-Gal4 and en-Gal4, and are being currently used for RNAi-based genetic screens for new determinants of $\mathrm{ChO}$ development (Hassan et al., unpublished data). ato-gal4 (Hassan et al., 2000) is a ChO-specific driver whose expression is restricted to the LCh5 lineage (Halachmi et al., 2016). It is expressed in the ChO precursors from stage 9 to 11 only, but the Gal4 protein persists in all of the cells derived from the ChO precursor, namely the CA, cap, scolopale, neuron and ligament cells. The expression of en-Gal4 (Brand and Perrimon, 1993), which is restricted to the posterior compartment of each segment, begins earlier, at the cellular blastoderm stage, and con-
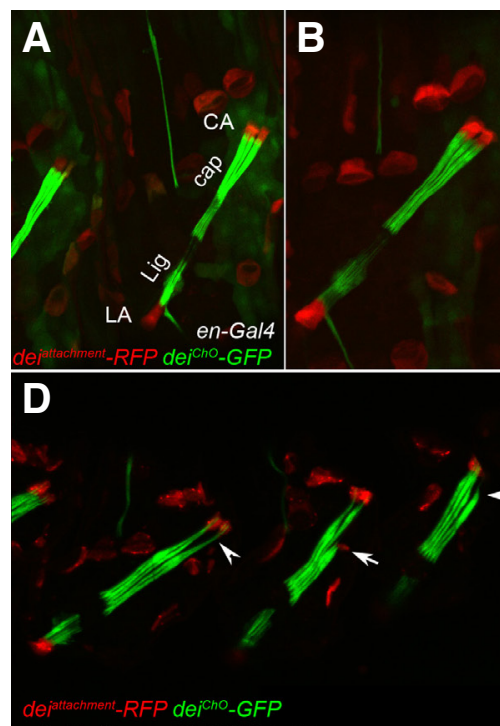

ato-Gal4>mys-IR HMSO0043

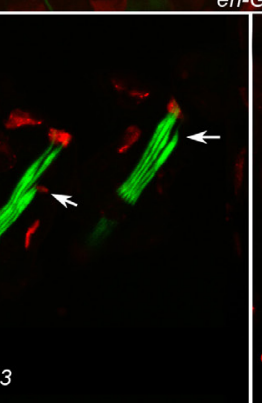

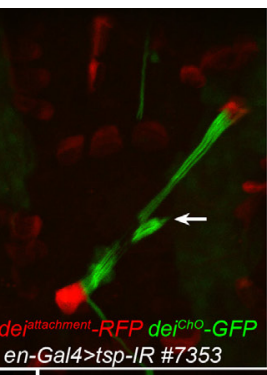
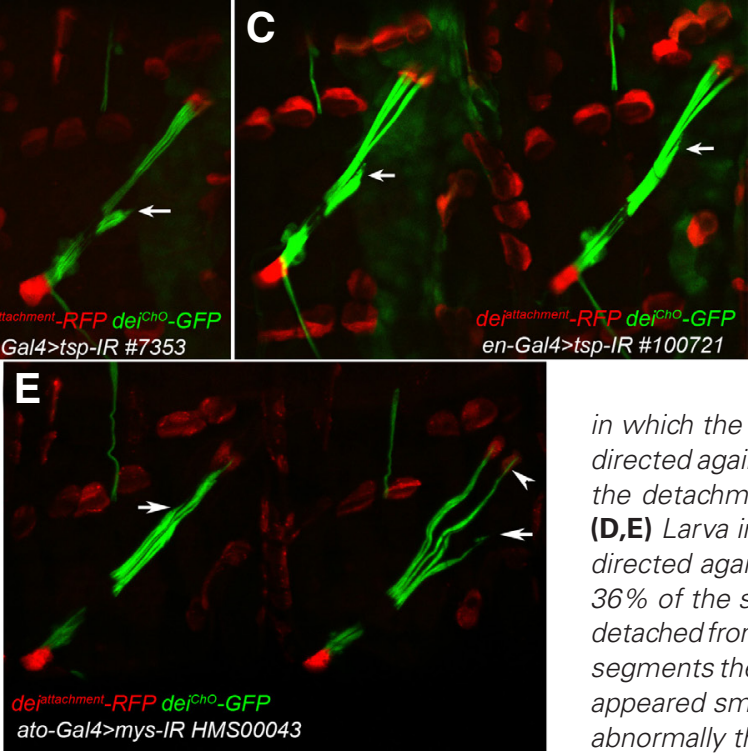

Fig. 1. The attachment of chordotonal organs (ChOs) to their attachment cells depends on thrombospondin (Tsp) and $\beta P S$ integrin. The LCh5 organs of second instar larvae as visualized by the

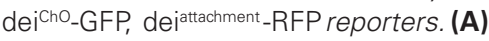
A control larva carrying the deicho-GFP, deiattachment -RFP chromosome and the en-gal4 driver. The CA, cap, ligament (Lig) and $L A$ cells are indicated. (B,C) Larvae in which the expression of two different RNAi constructs directed against the tsp gene were driven by en-gal4. Note the detachment of cap cells from the CA cells (arrows). (D,E) Larva in which the expression of an RNAi construct directed against the mys gene was driven by ato-gal4. In $36 \%$ of the segments ( $n=47)$ some of the cap cells were detached from the CA cells (arrows). In additional $57 \%$ of the segments the contact region between the cap and CA cells appeared smaller than normal and the cap cells appeared abnormally thin near the attachment site (arrowheads). 

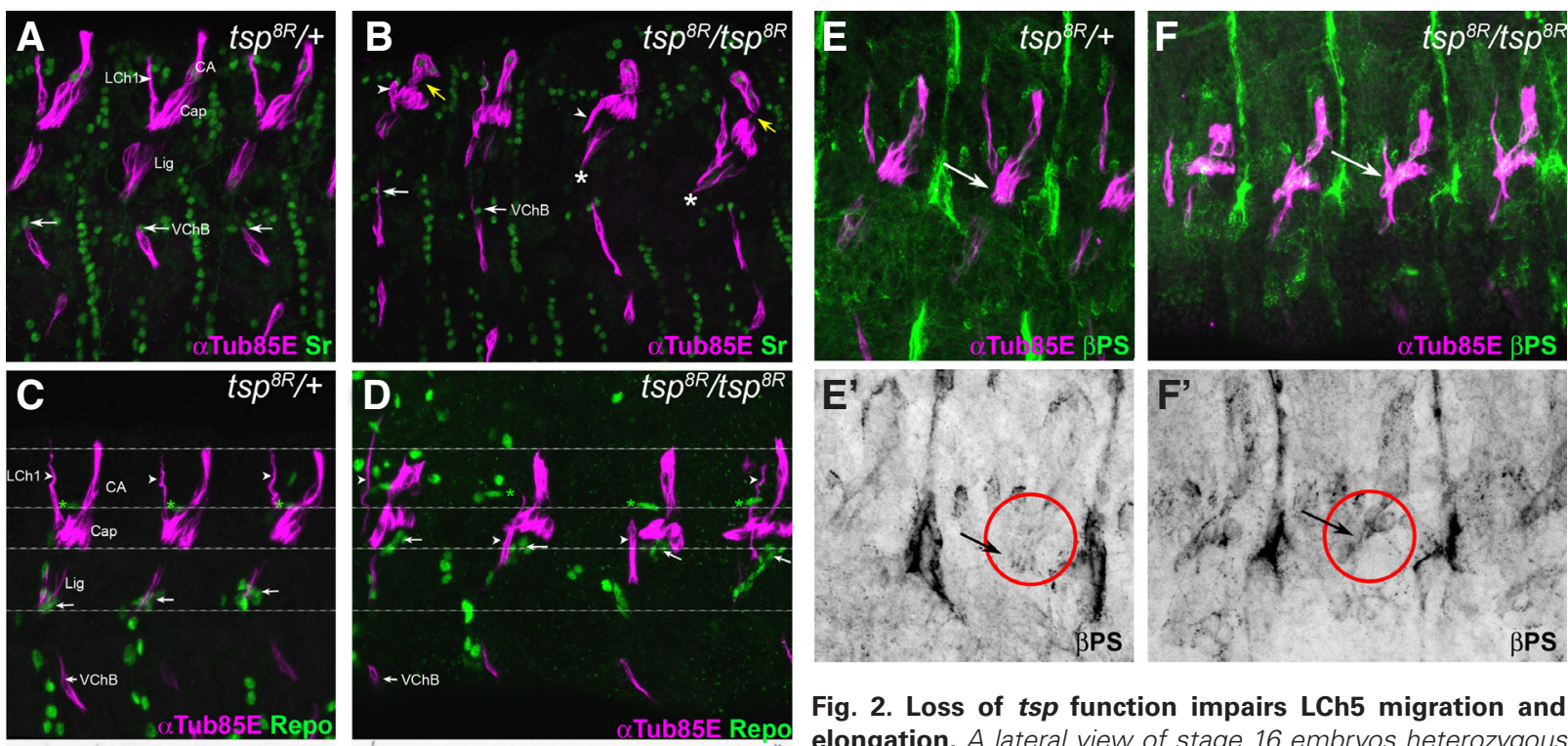

Fig. 2. Loss of tsp function impairs LCh5 migration and cap cell elongation. A lateral view of stage 16 embryos heterozygous (A, C, E)
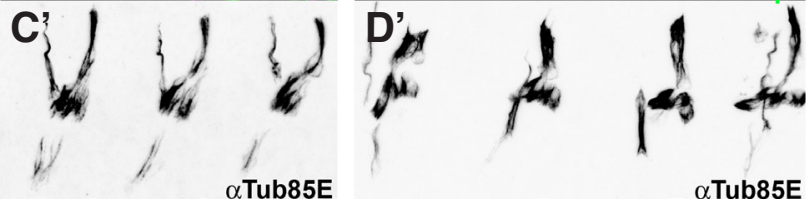

or homozygous (B, D, F) for the tsp ${ }^{8 \mathrm{R}}$ mutant allele. The tsp ${ }^{8 \mathrm{R}}$ chromosome was balanced over $\mathrm{CyO}$, wg-lacZ and embryos were stained with anti- $\beta$-gal (not shown) for unambiguous identification of their genotype. (A,B) Embryos stained for $\alpha$ Tub85E (magenta) and Sr (green). Defective attachment of cap-to-CA cells is evident in the mutant (yellow arrows in $B$ ). The asterisks in B mark the ventral tip of ligament cells that migrated relatively normally. Arrows point to cap cells of VChB organs that display an abnormal pattern of migration in the mutant, crossing the ventral tendons of the lateral transverse muscles. Arrowheads point to the cap cells of the LCh1 organ that display an abnormal migration pattern in the mutant. (C,D) Embryos stained for $\alpha$ Tub85E (magenta) and Repo (green). Arrowheads point to the cap cells of the LCh1 organ; Arrows point to the nuclei of the LCh5 ligament cells. (C'-D') The channel of $\alpha$ Tub85E staining is shown separately (inversed). (E,F) Embryos stained for $\alpha$ Tub85E and $\beta P S$ integrin. (E'-F') The $\beta P S$ integrin staining is shown separately. The arrows in $E-F$ and $E^{\prime}-F^{\prime}$ point to the ventral edge of the cap cells, which are encircled in red ( $\left.E^{\prime}-F^{\prime}\right)$. Note the abnormal distribution of $\beta P S$ integrin in the mutants.

tinues throughout embryonic development. The LCh5 organs are the only sensory organs originating in the posterior compartment (Hartenstein, 1987). Thus, the en-Gal4 driver drives continuous gene expression within the LCh5 lineage throughout its development, in addition to a stripe of ectodermal cells representing the posterior compartment.

One group of genes to be screened for their possible involvement in the attachment of $\mathrm{ChO}$ cells consists of ECM-related genes. To test the feasibility of the planned screen we have conducted a pilot screen in which $\sim 120$ strains harboring RNAi constructs directed against 64 ECM-related genes were expressed under the regulation of en-Gal4. Interestingly, the pilot screen revealed phenotypes that were not previously documented, and uncovered the importance of tsp in $\mathrm{ChO}$ morphogenesis and attachment.

Two strains harboring tsp-specific inverted repeat constructs were tested and both of them caused detachment of cap cells, most often of the two posterior cap cells, from their attachment cell when expressed under the regulation of en-gal4 (Fig. 1 B-C). Even though the penetrance of the detachment phenotype was quite low $(7.7 \%$ of segments, $n=350 ; 4.8 \%$ of segments, $n=371$, respectively) it appeared significant as both of the RNAi strains caused an identical phenotype that was not evident in the crosses done with any of the other 120 RNAi strains tested. These observations suggest that Tsp is required for the ability of the ChOs to maintain organ integrity in the larval stages when they are exposed to mechanical stresses imposed on them by muscle contractions. Since the en-gal driver does not drive expression in the LA cell, which is not derived from the ChO lineage (Inbal et al., 2004), we could not conclude from the results of this experiment about the possible involvement of Tsp in the attachment of ligament cells.

Since Tsp was implicated as a ligand for integrin receptors in the formation of the myotendinous junction (Subramanian et al., 2007), we tested for the involvement of integrin receptors in the formation of the ChO-attachment junction. $\beta \mathrm{PS}$-integrin expression was down-regulated within the $\mathrm{ChO}$ lineage by means of expressing a mys-specific RNAi transgene under the regulation of ato-gal4. The knockdown of integrin led to defects in the cap-to-CA attachment in $93 \%$ of the examined segments ( $n=47$ ) (Fig. 1 D-E). 36\% of the segments exhibited full detachment of the cap cells from the CA cells, similarly to the effect of tsp knockdown, supporting a possible interaction between Tsp and integrins in the formation of ChO attachment junctions.

\section{Loss of tsp prevents cap cell elongation and impairs ligament cell terminal differentiation and migration}

To establish whether Tsp is required only for the maintenance of the cap-to-CA connection, or is also required for its primary establishment, we have analyzed the phenotype of tsp mutant embryos. We found that $t s p^{8 R}$ homozygous embryos exhibited severe $\mathrm{ChO}$ phenotypes that extended beyond the attachment phenotypes seen in the tsp knockdown larvae. The most prominent defect evident in the ChOs of $t s p^{8 R}$ homozygous embryos was the inability of the LCh5 cap cells to elongate. In addition, the migration of the LCh1 and VChB cap cells was aberrant (Fig. 2 A-B). The collapse of 
the LCh5 cap cells could be the result of the detachment of these cells from the CA cells, to which they attach on their dorsal side, or an inability to properly fasten themselves to the scolopale cells, to which they attach to on their ventral side. Another possibility is that the cap cells fail to elongate due to a failure of the ligament cells to migrate ventrally and pull the entire organ with them. Anti-Repo staining, which labels the nuclei of the ligament cells, demonstrated a significant defect in ligament cell migration in $86.6 \%$ of the examined segments ( $\mathrm{N}=67$; Fig. $2 \mathrm{C}-\mathrm{D})$. In addition to defects in cell migration, the ligament cells of tsp mutant embryos displayed a reduction in the level of $\alpha$ Tub85E expression, suggesting defects in terminal differentiation. The aberrant pattern of ligament cell migration is most likely a major cause for the lack of cap cell stretching, however, the evident defects in the attachment of cap cells to the CA cells probably contribute to this phenotype as well. Furthermore, even in the $\sim 14 \%$ of segments in which ligament cell migration occurred relatively normally, the cap cells were not elongated and appeared detached from the CA cells (See asterisks at Fig. 2B).

As Tsp was shown to affect integrin localization and promote its accumulation at the leading edge of the myotube (Subramanian et al., 2007), a process required for initiating the formation of myotendinous junction, we tested the effect of tsp loss-offunction on integrin localization within the $\mathrm{ChO}$ cells. We found that similarly to the effect seen in muscle cells, loss of tsp leads to abnormal patterning of integrin distribution along the cap cells (Fig. 2 E-F). Although the role of integrins in cap cell elongation is not known, the above observations suggest that cap cells elongation is dependent on the normal composition and/or organization of the ECM and that this ability is affected by the loss of tsp function which alters integrin localization.

\section{Distribution of the Tsp protein in the ChO changes with organ maturation}

The embryonic expression pattern of Tsp has been previously described (Chanana et al., 2007), but no expression was reported in any of the $\mathrm{ChO}$ cells. The $\mathrm{ChO}$ phenotypes evident in tsp mutant embryos, and in tsp knockdown larvae, prompted us to re-examine the embryonic pattern of tsp transcription and to examine embryos and larvae for the presence of Tsp in the ECM surrounding the $\mathrm{ChO}$ cells. In situ hybridization with a tsp-specific probe revealed that in young embryos (stage 12-13), high levels of tsp mRNA are transcribed along the segment border. tsp mRNA was also evident in the posterior dorsolateral region of the segment where the LCh5 organs originate and start their migration (Fig. 3 A-B). In mature embryos (stage 17), tsp mRNA becomes evident in the $\mathrm{ChO}$ attachment cells (CA and $\mathrm{LA}$ ) and in the tendon cells (Fig. 3C). We could not determine unambiguously, based on the in situ hybridization, whether the ChO cap cells express low levels of tsp. Immuno-staining of embryos with anti-Tsp antibodies demonstrated accumulation of the Tsp protein around the LA cell in stage 17 embryos (Fig. 3D). A small amount of the Tsp protein was also detected at the contact zone between the cap and CA cell at this stage.

In the larva, Tsp accumulates at very high levels in the cap/ $\mathrm{CA}$ - and in the ligament/LA cell junctions (Fig. 4 A-C). The high levels of Tsp, as well as integrin, integrin-linked kinas (ILK)-GFP and Talin-GFP (Fig. 4 and data not shown), suggest that the junctions between the cap and CA cells and between the ligament and LA cells resemble the myotendinous junction in the repertoire of molecules that constitutes them. In addition to the accumulation of high levels of Tsp at the anchoring sites of the ChO to its attachment cells, the Tsp protein was also found to decorate the cap cells along their entire length (Fig. 4 D-E). Punctate staining was also seen around the scolopale and ligament cells (Fig. 4C). The levels of Tsp accumulation along the cap cells were much lower than the levels accumulated at the distal attachment site and the distribution of the protein appeared patchy (Fig. 4 DE). Examination of $\beta$ PS integrin distribution, as well as ILK-GFP, a hallmark of integrin activity at hemiadherens junctions, revealed their presence along the entire length of the cap cells in addition to their high concentration at the distal attachment sites (Fig. 4 E-F and data not shown). These observations suggest that the elongated cap cells are supported by the ECM, to which they attach along their entire length via integrin-based, Tsp-dependent, adhesion plaques.

The role of this lateral cell-ECM connection is not known.
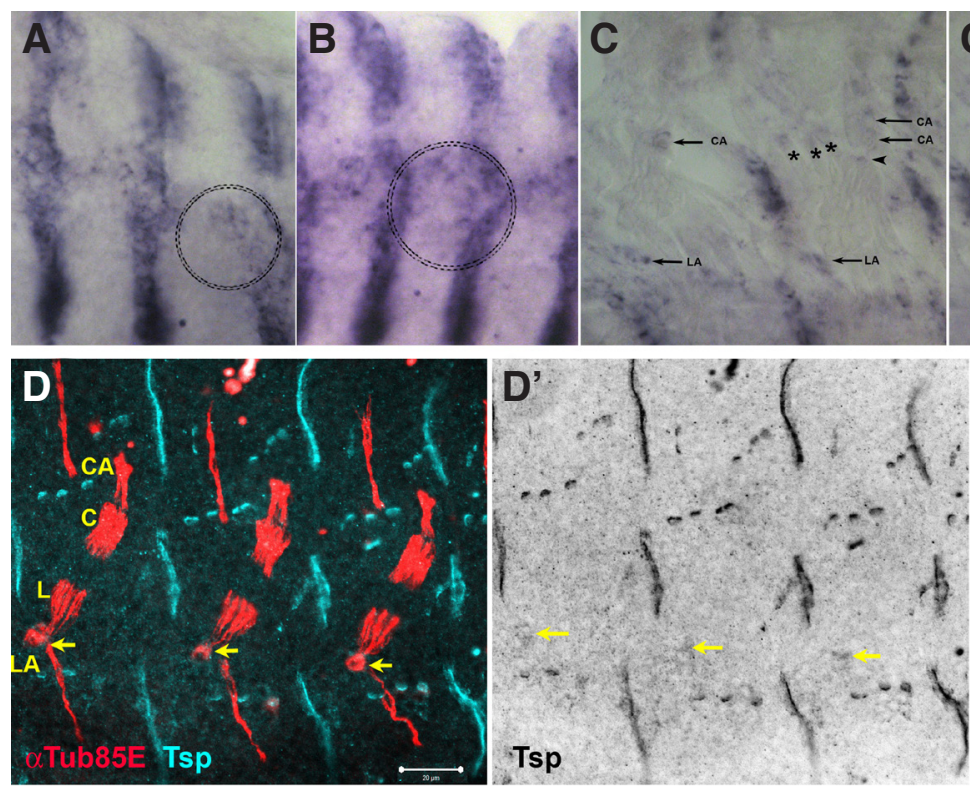

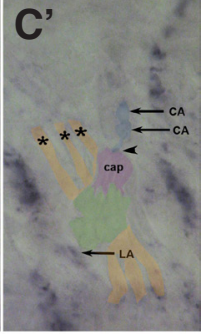

Fig. 3. Embryonic expression pattern of the tsp gene. (A-C) In situ hybridization with a tsp-specific probe to stage 12 (A), 13 (B) and 17 (C) embryos. The circles in $\mathbf{( A , B )}$ mark the location of the LCh5 organ at these developmental stages. (C) Two segments of a stage 17 embryo in which the LCh5 can be identified with Nomarski optics. The arrows point to the CA and LA cells; the arrowhead points to the attachment site between the cap and CA cells; the asterisks mark the lateral transverse muscles. A duplicate of the right segment is shown in (C') where the CA cells are highlighted in blue, the cap cells are highlighted in purple, the LCh5 sensory unit is highlighted in green and the lateral transverse muscles are highlighted in yellow. (D,D') A stage 17 embryo

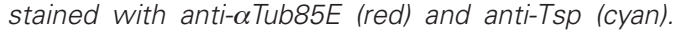
The Tsp staining is shown separately in ( $\left.\mathbf{D}^{\prime}\right)$. The yellow arrows point to the LA cells. 

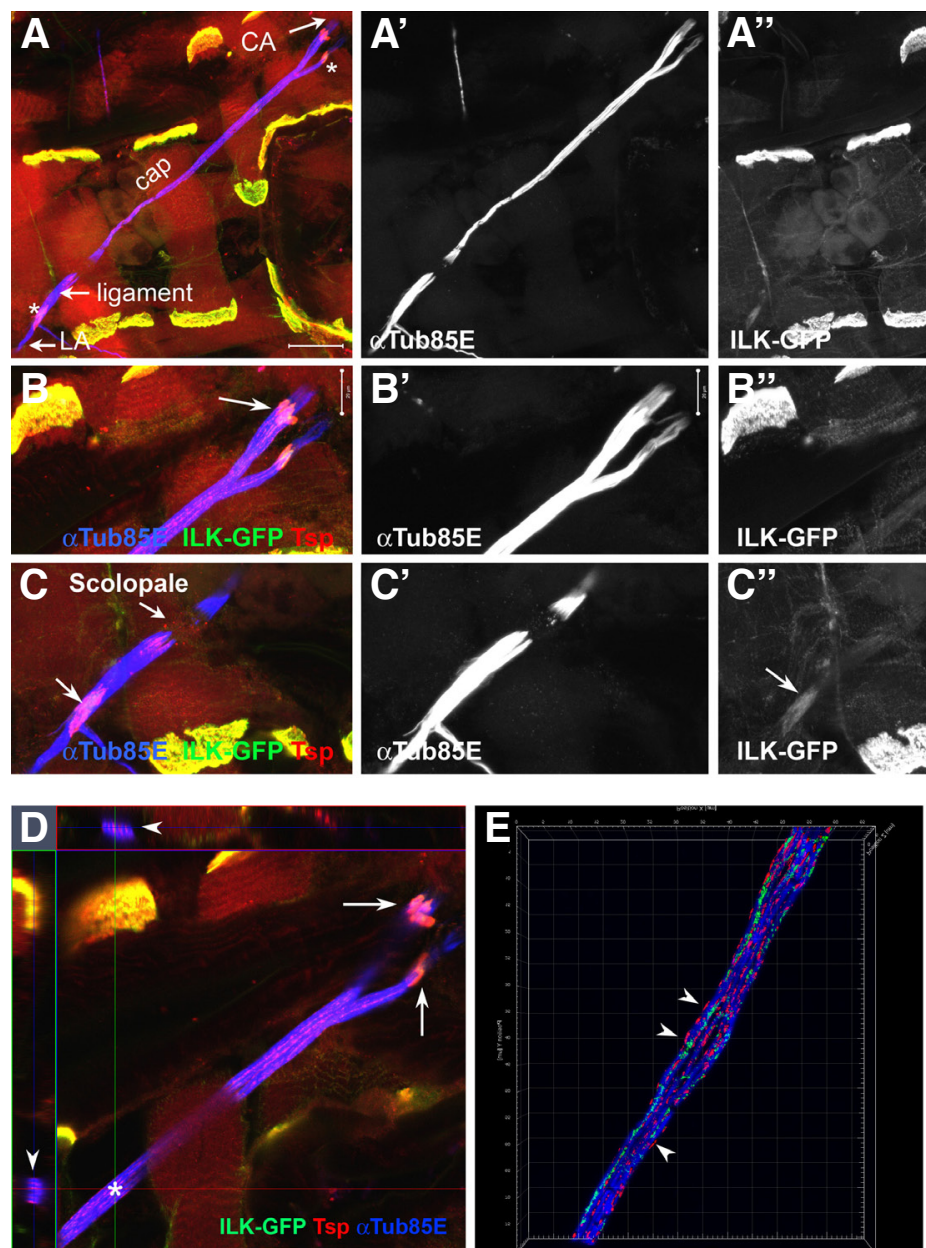

Unfortunately, it could not be deduced from the analysis of tsp or integrin knockdown experiments in which the RNAi transgenes were driven by ato-gal4 or en-gal4. The reason is that the cap/ CA junction, which experiences strong mechanical stresses in the direction of the stretching axis, is the first to fail upon reduction of Tsp or integrin activity, thereby masking the possible involvement of tsp in supporting the cap cells themselves. To address the role of the lateral cap-ECM connection, we would need to interfere specifically with the lateral cell-ECM connection (along the cap cell) without disturbing the distal junctions (between the cap and CA cells). The genetic tools required for such a spatially restricted manipulation are currently not available.

\section{Embryonic phenotypes of tsp mutants point to a possible role of the lateral ECM support in cap cell elongation}

The EGR-like transcription factor $\mathrm{Sr}$ is a major determinant of tendon cell and $\mathrm{ChO}$ attachment cell development (Frommer et al., 1996; Inbal et al., 2004). It also plays a role in ligament cell differentiation (Klein et al., 2010) and was shown to induce tsp expression in epidermal cells when ectopically expressed (Subramanian et al., 2007). The abnormal ChO morphology of tsp mutant embryos is highly reminiscent of the phenotypes seen in srmutant embryos. In srmutants the LCh5 ligament cells fail to migrate properly and they present highly reduced levels of $\alpha$ Tub85E expression (Klein et al., 2010). However, in spite of the defects in ligament cell migration and in cap-to-CA attachment, the cap cells of sr mutant embryos
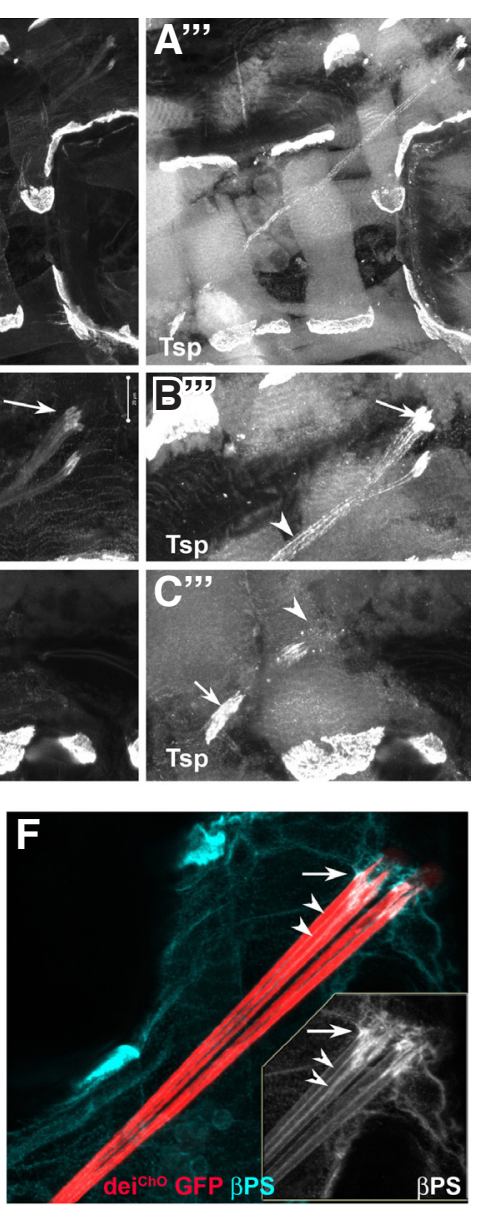

Fig. 4. Tsp and $\beta P S$ integrin accumulate at high levels in the $\mathrm{ChO}$ attachment sites and decorate the cap cells along their entire length in larval stages. (A-C) $A n$ LCh5 organ of an ILK-GFP third instar larva stained for $\alpha$ Tub85E (blue) and Tsp (red). The different channels are shown separately in $\left(A^{\prime}-A^{\prime \prime \prime}, B^{\prime}-B^{\prime \prime \prime}\right.$ $\left.C^{\prime}-C^{\prime \prime \prime}\right)$. (A) An overall view of the entire organ. (B) A close-up view of the cap-CA junction (arrow). (C) $A$ close-up via of the ligament-LA junction (arrow) and the scoloplae cell. (D) An optical section through the cap cells of the LCh5 organ shown in $B$, demonstrating the accumulation of Tsp around each of the five cap cells (arrowheads). (E) A $3 D$ reconstruction (using Imaris) of LCh5 Cap cells of an ILKGFP third instar larva stained for $\alpha$ Tub85E (blue) and Tsp (red). The arrowheads point to the patchy distribution ofTsp in the ECM surrounding the cells. The asterisks mark the position of the optical section (F) An LCh5 organ of a deicho GFP (GFP is shown in red) third instar larva stained for $\beta P S$-integrin (cyan). The $\beta P S$-integrin staining is shown separately in the inset. The arrows point to the accumulation of $\beta P S$-integrin at the cap/CA attachment site; arrowheads point to the distribution of $\beta P S$-integrin along the cap cells.

appear longer (less collapsed) than the cap cells of tsp mutants (compare Fig. 5B to Fig. 2B,D). This observation could suggest that the lateral support of the ECM enwrapping the cap cells is critical for cell elongation. It also suggests that the cap cell phenotypes of tsp mutants are, at least partially, sr-independent. Indeed, Sr is not expressed in the cap cells and thus it cannot regulate the transcription of $t s p$ or its receptor in these cells. Sr could however be affecting tsp transcription in the ChO attachment cells.

To examine the involvement of $\mathrm{Sr}$ in the regulation of $\mathrm{Tsp}$ deposition in the ECM of different ChO cells, we examined the pattern of Tsp distribution in $s r$ mutant embryos and in $3^{\text {rd }}$ instar larvae in which sr expression was reduced by RNAi expression in the ChO lineage (under ato-Gal4). As previously described, in the embryo, loss of $\mathrm{Sr}$ function led to loss of Tsp accumulation in the myotendinous junctions of the lateral transverse muscles and to less pronounced reduction in myotendinous junctions of other muscles (Fig. $5 \mathrm{~A}-\mathrm{B}$ ). The amount of Tsp protein in the cap/ $\mathrm{CA}$ junction in late embryogenesis is minuscule, but identifiable in most LCh5 organs of $s r$ heterozygous embryos. No such accumulation is evident in the LCh5 of srhomozygous mutants. The LA cell does not form in sr mutant embryos and accordingly, Tsp does not accumulate in this region (Fig. 5 A-B).

In third instar larvae, in which sr expression was knocked down specifically within the $\mathrm{ChO}$ lineage, the CA failed to differentiate properly, as indicated by the loss of the dejattachment reporter. The cap/CA junction did not form properly and the cap cells were often 
detached (Fig. 5 C-D). In accordance with that, Tsp did not accumulate at high levels around the cap cell distal tip in the cap/CA junction region. Nevertheless, normal levels of Tsp accumulated in the ECM enwrapping the cap cells (Fig. 5D). These results support our assumption that $\mathrm{Sr}$, which is not expressed in the cap cells, does not regulate the expression of Tsp, or its receptor, in these cells. It may also suggest that the Tsp protein present in the cap cell's lateral ECM does not originate in the CA cells. However, additional experiments are required for validating this notion, since we cannot know for sure whether the sr-RNAi fully eliminated $s r$ expression in the CA cells.

\section{Discussion}

Genetic screens provide a powerful means for identifying genes required for organogenesis or function of any system of choice, especially when a convenient phenotypic readout is available. Recently, we have generated fluorescent reporters that allow for easy phenotypic analyses of larval ChOs. We used these tools to test the feasibility of an RNAi screen aimed at identifying ECM components required for $\mathrm{ChO}$ morphogenesis. During the course
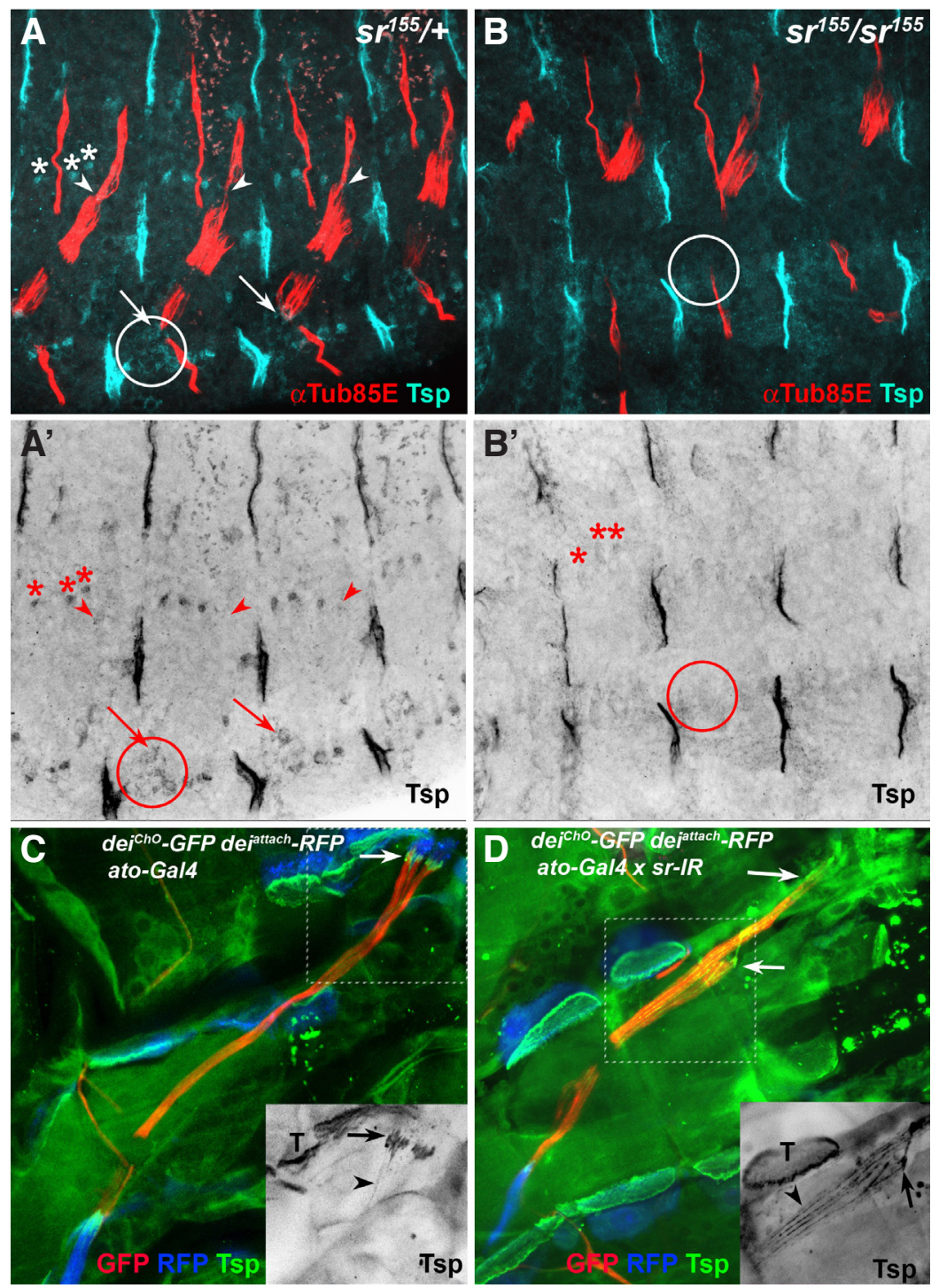

of the small pilot screen the tsp gene was identified. Tsp is a known player in the establishment of the myotendinous junctions in both invertebrates and vertebrates (Frolova et al., 2014; Subramanian et al., 2007; Subramanian and Schilling, 2014). Now, we show that it is also critical for the establishment and maintenance of the junction between the $\mathrm{ChO}$ and its attachment cells. Our findings extend the known similarities between muscle-attachment and ChO-attachment cells and highlight the similar repertoire of molecules that constitute the attachment junctions in both systems.

The dynamics of Tsp accumulation at the ChO-specificjunction is different than the dynamics observed at the muscle-tendon system. In early embryonic stages, prior to the formation of muscle-tendon junctions, Tsp is detected in a punctate pattern around the Sr-positive epidermal attachment cells that will eventually differentiate as tendon cells. During late embryogenesis, following the establishment of the myotendinous junction, Tsp becomes highly concentrated at the muscle-tendon junction sites (Subramanian et al., 2007). In the ChO, Tsp is first detected during late embryogenesis around the Sr-positive LA cell. Then, towards the end of embryogenesis, very low levels of Tsp become evident at the attachment site of the cap cells to their attachment cells. It is only later, in larval stages, with the increase in mechanical strains and the significant growth of the $\mathrm{ChO}$ attachment cells, that Tsp accumulates at very high levels (comparable to the levels seen in myotendinous junctions) at the cap/CA and ligament/LA attachment points. This accumulation of Tsp coincides with the accumulation of high levels of integrins, ILK, and Talin, pointing to structural similarities with the myotendinous hemiadherens junction.

Although we could not clearly detect Tsp accumulation around the cap and CA cells during embryogenesis, the phenotypes caused by tsp loss-of-function strongly suggest that it is present in the ECM surrounding these cells and that it is required for the formation of cap/CA junctions and for cap cell elongation. It is possible that Tsp is present in the ECM surrounding these cells in very low levels that are below the threshold of detection. In addition, the abundance of low levels of Tsp in neighboring tissues at this stage could mask its presence in the ChO cells' ECM. The idea that Tsp is required for cap cell elongation and that the cap cells interact with the

Fig. 5. The accumulation of thrombospondin (Tsp) along the cap cells is Sr-independent. (A,B) Stage 17 embryos, heterozygous (A) or homozygous (B) for the $\mathrm{sr}^{155}$ allele, stained for $\boldsymbol{\alpha}$ Tub85E (red) and Tsp (cyan). Tsp expression is shown separately in $\left(A^{\prime} B^{\prime}\right)$. Tsp accumulation at the attachment sites of the lateral transverse muscles is indicated with asterisks in the heterozygous embryo (A); such accumulation is not evident in the mutant (B). The arrowheads point to the cap-CA attachment site; the arrows point to the ligament/LA attachment site. The patch of epidermal Tsp expression neighboring the $L A$ cell is encircled in (A). The corresponding region lacking such expression is encircled in (B). (C,D) LCh5 organs of third instar deicho-GFP - dejattachment-RFP, ato-gal4 (A) and deicho_GFP, dejattachment_RFP, ato-gal4/sr-IR (B) larvae stained with anti-Tsp (green). GFP is shown in red and RFP in blue; Tsp expression is shown separately in the inset. The arrows point to the cap-CA attachment sites. The arrowheads point to the localization of Tsp along the cap cells. T, tendon cells. 
ECM along their entire length already during embryogenesis is supported by the presence of $\beta$ PS integrin along these cells and the abnormal pattern of $\beta$ PS-integrin distribution evident in the cap cells of tsp mutant embryos.

In larval stages, the accumulation of Tsp and other ECM components around the cap cells, which increase in length more than ten fold, becomes much more pronounced. This ECM wrap around these cells is probably important for keeping the five cap cells fasciculate and may also provide a mechanical support that allows the extremely elongated cells to maintain tension. Whether Tsp is produced by the cap cells themselves, or is produced by neighboring cells and recruited to the cap cell's ECM, is a question we still have to answer.

Another unexpected effect of tsp loss-of-function is the impairment of ligament cell migration. Very little is known about the cues guiding the ligament cells in their migration and about the identity of the guiding posts along their migratory path. The aberrant ligament cell migration is likely independent of the muscle phenotype of $t s p$ mutants. One reason is that ligament cells migrate independently of the tendons that direct muscle cells in their migration (Klein et al., 2010). The other reason is that muscles develop normally in early tsp mutant embryos and the muscle phenotypes become evident only in late embryogenesis (Subramanian et al., 2007), whereas ligament cell migration takes place during earlier embryonic stages. It is possible that Tsp is required for correct localization or accumulation of signaling molecules at certain segmental positions that serve as guiding posts for the migrating ligament cells. It is also possible that Tsp directly affects the ECM of the migrating ligament cells. Numerous studies in vertebrates have demonstrated the involvement of Tsp molecules in cell migration in normal development and under pathological conditions (e.g. (Adams and Lawler, 2011; Girard et al., 2014).

Altogether, this work unravels the involvement of Tsp in cell migration, differentiation and attachment of the $\mathrm{ChO}$ and provides an entry point for studying the role of the ECM in patterning and functioning of proprioceptive sensory organs.

\section{Materials and Methods}

\section{Fly strains}

The following fly strains were used in this study (described in FlyBase http://flybase.bio.indiana.edu/ (St Pierre et al., 2014) unless otherwise indicated): dejcho_GFP, dejattachment_RFP (Halachmi et al., 2016), Il $k^{z C L 311}$, ato-gal4 (Hassan et al., 2000), en-Gal4, tsp ${ }^{8 R}$ is an embryonic lethal small deletion allele of $t s p$ (Subramanian et al., 2007), sri55 (Usui et al., 2004). The tsp-IR strains $P\{G D 485\} v 7535$ and $P\{K K 108103\} v 100721$ are viable strains on the $3^{\text {rd }}$ and $2^{\text {nd }}$ chromosome, respectively, with no off target sequences. The $P\{K K 107677\}$ V105282 RNAi strain was used for knocking down sr expression. P\{TRiP.HMS00043\}attp2 was used to knock down mys ( $\beta P S$-integrin) expression. The RNAi strains were obtained from the Vienna Drosophila Resource Center (Dietzl etal., 2007) and the Bloomington Drosophila Stock Center (http://flystocks.bio.indiana.edu).

\section{In situ hybridization}

A $551 \mathrm{bp}$ fragment of $t s p$ coding sequence was amplified by PCR, from a cDNA template, using the following primers: 5' GATACCCTTGCGACGTGTTT 3' and 5' GGTCTAATGTGTCGCGTTGA3'. This fragment was used as a template for synthesizing sense and anti-sense digoxigenin-labeled riboprobes. Whole-mount insitu hybridization was carried out as described by Krause and colleagues (Wilk et al., 2010).

\section{Immunohistochemistry}

Immuno-staining of whole-mount embryos was performed using standard techniques. Immuno-staining of third instar larvae was carried out as described in (Halachmi et al., 2012). For phenotypic analysis of whole-mount $2^{\text {nd }}$ instar larvae, larvae were washed once with phosphate buffered saline $+0.1 \%$ Tween-20 (PBT) and fixed over night at $4{ }^{\circ} \mathrm{C}$ in $4 \%$ formaldehyde in PBT. Fixed larvae were washed twice with PBT (over 20 minutes) and twice with PBS (over 20 minutes) before mounting. Stained embryos and larvae were mounted in Dako Fluorescent Mounting Medium (DakoCytomation, Glostrup, Denmark) and viewed using confocal microscopy (LSM 510, Zeiss). Primary antibodies used in this study: Guinea pig anti SrA/B (1:300, (Becker et al., 1997), chicken anti- $\beta$-galactosidase (1:400, abcam), rabbit and mouse anti- $\alpha$ Tub-85E (Klein et al., 2010; Nachman et al., 2015,

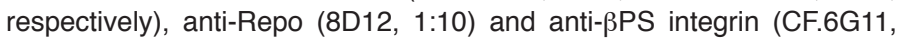
concentrated 1:100), were obtained from the Developmental Studies Hybridoma Bank, created by the NICHD of the NIH and maintained at The University of lowa, Department of Biology, lowa City, IA 52242. Secondary antibodies for fluorescent staining were Cy5, Cy2 or Alexa 647conjugated anti mouse/rabbit/guinea pig/chicken (Jackson Immunoresearch Laboratories, Bar-Harbor, Maine, USA).

\section{Acknowledgements}

We wish to thank T. Volk, D. Ben-Zvi, L. Gilboa, E. Kurant, the Bloomington Stock Center, the Vienna Drosophila Resource Center and the Developmental Studies Hybridoma Bank at the University of lowa for plasmids, antibodies and fly strains. This work was supported by a grant (No. 499/12) from The Israel Science Foundation.

\section{References}

ADAMS JC, LAWLER J (2011). The thrombospondins. Cold Spring Harb Perspect Biol 3: a009712.

ADAMS JC, MONK R, TAYLOR AL, OZBEK S, FASCETTI N, BAUMGARTNER S, ENGELJ (2003). Characterisation of Drosophilathrombospondin defines an early origin of pentameric thrombospondins. J Mol Biol 328: 479-494.

ADOLPH KW (2001). A thrombospondin homologue in Drosophila melanogaster: cDNA and protein structure. Gene 269: 177-184.

BECKER S, PASCA G, STRUMPF D, MIN L, VOLK T (1997). Reciprocal signaling between Drosophila epidermal muscle attachment cells and their corresponding muscles. Development 124: 2615-2622.

BRAND AH, PERRIMON N (1993). Targeted gene expression as a means of altering cell fates and generating dominant phenotypes. Development 118: 401-415.

BREWSTERR, BODMER R(1995). Origin and specification of type ll sensory neurons in Drosophila. Development 121: 2923-2936.

CALDWELL JC, MILLER MM, WING S, SOLL DR, EBERL DF (2003). Dynamic analysis of larval locomotion in Drosophila chordotonal organ mutants. Proc Natl Acad Sci USA 100: 16053-16058.

CHANANA B, GRAF R, KOLEDACHKINA T, PFLANZ R, VORBRUGGEN G (2007). AlphaPS2 integrin-mediated muscle attachment in Drosophila requires the ECM protein Thrombospondin. Mech Dev 124: 463-475.

DIETZL G, CHEN D, SCHNORRER F, SU K-C, BARINOVAY, FELLNER M, GASSER B, KINSEY K, OPPEL S, SCHEIBLAUER S, COUTO A, MARRA V, KELEMAN K, DICKSON BJ (2007). A genome-wide transgenic RNAi library for conditional gene inactivation in Drosophila. Nature 448: 151-156.

FROLOVA EG, DRAZBA J, KRUKOVETS I, KOSTENKO V, BLECH L, HARRY C VASANJI A, DRUMM C, SUL P, JENNISKENS GJ, PLOW EF, STENINAADOGNRAVI O (2014). Control of organization and function of muscle and tendon by hrombospondin-4. Matrix Biol 37: 35-48.

FROMMER G, VORBRUGGEN G, PASCA G, JACKLE H, VOLKT (1996). Epidermal egr-like zinc finger protein of Drosophila participates in myotube guidance. EMBO J 15: 1642-1649.

GHYSEN A, DAMBLY-CHAUDIERE C (1989). Genesis of the Drosophila peripheral nervous system. Trends Genet 5: 251-255. 


\section{R. T. Greenblatt et al.}

GIRARD F, EICHENBERGER S, CELIO MR (2014). Thrombospondin 4 deficiency in mouse impairs neuronal migration in the early postnatal and adult brain. $\mathrm{Mol}$ Cell Neurosci 61: 176-186.

GONG Z, SON W, CHUNG YD, KIM J, SHIN DW, MCCLUNG CA, LEE Y, LEE HW, CHANG D-J, KAANG B-K, CHO H, OH U, HIRSH J, KERNAN MJ, KIM C (2004). Two interdependent TRPV channel subunits, inactive and Nanchung, mediate hearing in Drosophila. J Neurosci 24: 9059-9066.

HALACHMI N, NACHMANA, SALZBERGA(2016). Anewly identified type of attachment cell is critical for normal patterning of chordotonal neurons. Dev Biol 411: 61-71.

HALACHMI N, NACHMAN A, SALZBERG A (2012). Visualization of proprioceptors in Drosophila larvae and pupae. J Vis Exp: e3846.

HARTENSTEIN V (1987). The influence of segmental compartmentalisation on the development of the larval peripheral nervous system in Drosophila melanogaster. Roux's Arch Dev Biol 196: 101-112.

HASSAN BA, BERMINGHAM NA, HE Y, SUN Y, JAN YN, ZOGHBI HY, BELLEN HJ (2000). atonal regulates neurite arborization but does not act as a proneural gene in the Drosophila brain. Neuron 25: 549-561.

HASSON P, VOLK T, SALZBERG A (2017) Building functional units of movementgeneration and movement-sensation in the embryo. Int J Dev Biol. 61: 171-178.

INBALA, LEVANON D, SALZBERG A(2003). Multiple roles for u-turn/ventral veinless in the development of Drosophila PNS. Development 130: 2467-2478.

INBALA, VOLK T, SALZBERG A (2004). Recruitment of ectodermal attachment cells via an EGFR-dependent mechanism during the organogenesis of Drosophila proprioceptors. Dev Cell 7: 241-250.

JARMAN AP, GRAU Y, JAN LY, JAN YN (1993). atonal is a proneural gene that directs chordotonal organ formation in the Drosophila peripheral nervous system. Cell 73: 1307-1321.

KIM J, CHUNG YD, PARK D-Y, CHOI S, SHIN DW, SOH H, LEE HW, SON W, YIM
J, PARK C-S, KERNAN MJ, KIM C (2003). A TRPV family ion channel required for hearing in Drosophila. Nature 424: 81-84

KLEIN Y, HALACHMI N, EGOZ-MATIA N, TODER M, SALZBERG A (2010). The proprioceptive and contractile systems in Drosophila are both patterned by the EGR family transcription factor Stripe. Dev Biol 337: 458-470.

MATTHEWS KA, MILLER DF, KAUFMAN TC (1990). Functional implications of the unusual spatial distribution of a minor alpha-tubulin isotype in Drosophila: a common thread among chordotonal ligaments, developing muscle, and testis cyst cells. Dev Biol 137: 171-183.

NACHMAN A, HALACHMI N, MATIA N, MANZUR D, SALZBERG A (2015). Deconstructing the complexity of regulating common properties in different cell types: lessons from the delilah gene. Dev Biol 403: 180-191.

PROKOP A, MARTÍN-BERMUDO MD, BATE M, BROWN NH (1998). Absence of $P S$ integrins or laminin A affects extracellular adhesion, but not intracellular assembly, of hemiadherens and neuromuscular junctions in Drosophila embryos. Dev Biol 196: 58-76.

ST PIERRE SE, PONTING L, STEFANCSIK R, MCQUILTON P, FLYBASE C (2014). FlyBase 102--advanced approaches to interrogating FlyBase. Nucleic Acids Res 42: D780-788.

SUBRAMANIAN A, SCHILLING TF (2014). Thrombospondin-4 controls matrix assembly during development and repair of myotendinous junctions. Elife $3: \mathrm{e} 02372$

SUBRAMANIAN A, WAYBURN B, BUNCH T, VOLK T (2007). Thrombospondinmediated adhesion is essential for the formation of the myotendinous junction in Drosophila. Development 134: 1269-1278.

USUI K, PISTILLO D, SIMPSON P (2004). Mutual exclusion of sensory bristles and tendons on the notum of dipteran flies. Curr Biol 14: 1047-1055.

WILK R, MURTHY S U, YAN H, KRAUSE H M (2010). In situ Hybridization: Fruit Fly Embryos and Tissues. Curr. Protocol. Essent. Lab. Tech. 4:9.3:9.3.1-9.3.24. 


\section{Further Related Reading, published previously in the Int. J. Dev. Biol.}

The involvement of three signal transduction pathways in botryllid ascidian astogeny, as revealed by expression patterns of representative genes

Amalia Rosner, Gilad Alfassi, Elizabeth Moiseeva, Guy Paz, Claudette Rabinowitz, Ziva Lapidot, Jacob Douek, Abraham Haim and Baruch Rinkevich

Int. J. Dev. Biol. (2014) 58: 677-692

Mouse models to study inner ear development and hereditary hearing loss Lilach M. Friedman, Amiel A. Dror and Karen B. Avraham Int. J. Dev. Biol. (2007) 51: 609-631

The importance of the posterior midline region for axis initiation at early stages of the avian embryo

Oded Khaner

Int. J. Dev. Biol. (2007) 51: 131-137

Early stages of neural crest ontogeny: formation and regulation of cell delamination Chaya Kalcheim and Tal Burstyn-Cohen Int. J. Dev. Biol. (2005) 49: 105-116

Allogeneic interactions in Hydractinia: is the transitory chimera beneficial? Sharon Gild, Uri Frank and Ofer Mokady

Int. J. Dev. Biol. (2003) 47: 433-438

High proliferation rate characterizes the site of axis formation in the avian blastula-stage embryo.

N Zahavi, V Reich and O Khaner

Int. J. Dev. Biol. (1998) 42: 95-98
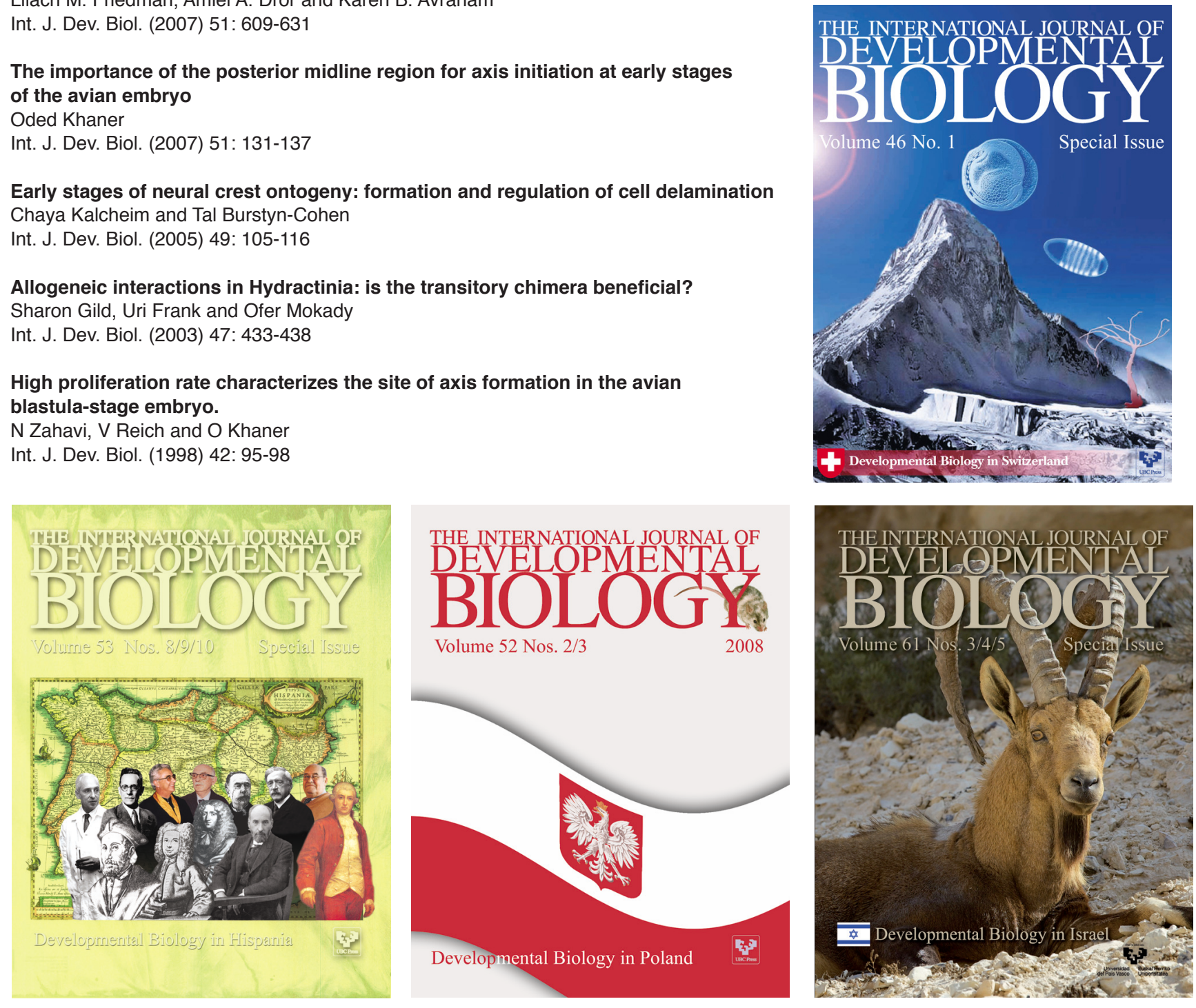